\title{
Transcriptome Analysis of Durusdinium Associated with the Transition from Free-Living to Symbiotic
}

\author{
Ikuko Yuyama ${ }^{1, *}$, Naoto Ugawa ${ }^{2}$ and Tetsuo Hashimoto ${ }^{2}$ \\ 1 Graduate School of Science and Technology for Innovation, Yamaguchi University, 1677-1 Yoshida, \\ Yamaguchi 753-8512, Japan \\ 2 Faculty of Life and Environmental Sciences, University of Tsukuba, 111 Tennodai, Tsukuba, \\ Ibaraki 305-8577, Japan; ugawa.naoto.sw@alumni.tsukuba.ac.jp (N.U.); \\ hashimoto.tetsuo.gm@u.tsukuba.ac.jp (T.H.) \\ * Correspondence: yuyamai@gmail.com; Tel.: +81-83-933-5835
}

check for updates

Citation: Yuyama, I.; Ugawa, N.; Hashimoto, T. Transcriptome Analysis of Durusdinium Associated with the Transition from Free-Living to Symbiotic. Microorganisms 2021, 9 , 1560. https://doi.org/10.3390/ microorganisms 9081560

Academic Editor: Shauna Murray

Received: 14 April 2021

Accepted: 12 July 2021

Published: 22 July 2021

Publisher's Note: MDPI stays neutral with regard to jurisdictional claims in published maps and institutional affiliations.

Copyright: (c) 2021 by the authors. Licensee MDPI, Basel, Switzerland. This article is an open access article distributed under the terms and conditions of the Creative Commons Attribution (CC BY) license (https:/ / creativecommons.org/licenses/by/ $4.0 /)$.

\begin{abstract}
To detect the change during coral-dinoflagellate endosymbiosis establishment, we compared transcriptome data derived from free-living and symbiotic Durusdinium, a coral symbiont genus. We detected differentially expressed genes (DEGs) using two statistical methods (edgeR using raw read data and the Student's $t$-test using bootstrap resampling read data) and detected 1214 DEGs between the symbiotic and free-living states, which we subjected to gene ontology (GO) analysis. Based on the representative GO terms and 50 DEGs with low false discovery rates, changes in Durusdinium during endosymbiosis were predicted. The expression of genes related to heat-shock proteins and microtubule-related proteins tended to decrease, and those of photosynthesis genes tended to increase. In addition, a phylogenetic analysis of dapdiamide A (antibiotics) synthase, which was upregulated among the 50 DEGs, confirmed that two genera in the Symbiodiniaceae family, Durusdinium and Symbiodinium, retain dapdiamide A synthase. This antibiotic synthaserelated gene may contribute to the high stress tolerance documented in Durusdinium species, and its increased expression during endosymbiosis suggests increased antibacterial activity within the symbiotic complex.
\end{abstract}

Keywords: RNA-seq; scleractinian coral; Symbiodiniaceae; endosymbiosis

\section{Introduction}

The dinoflagellate of the Symbiodiniaceae family live symbiotically with a variety of marine invertebrates, including clams, sea slugs, sea anemones, foraminifera, and corals [1-3]. Among these, the symbiotic relationships between symbiodiniacean algae and cnidarians have been studied extensively. Symbiotic algae provide photosynthetic products to corals and receive nitrogen in exchange [4-6]. Published evidence indicates that the activity of symbiotic Symbiodiniaceae is under the control of the host corals [7-9]. In coral cells, algae are present in host-derived acidified vesicles that have carbon-concentrating mechanisms and activate photosynthetic capacity [7]. A recent transcriptome analysis found that dinoflagellate genes involved in molecular chaperoning as well as sugar and ammonia transportation were suppressed during the establishment of endosymbiosis with Aiptasia and coral planula larvae [10,11]. Gene expression analyses of actin, $\mathrm{Ca}^{2+}$ ATPase, and $\mathrm{H}^{+}$APTase in Symbiodiniaceae also revealed that their expression patterns differed considerably between the non-symbiotic and symbiotic states [12,13]. However, despite these recent advances, many aspects of the changes dinoflagellate undergo during coral endosymbiosis establishment remain unclear. Previous studies have established a model endosymbiosis system consisting of monoclonal alga and juvenile corals, and transcriptome data for these coral-alga complexes have been published, with a focus on coral gene expression [11,14-16]. By contrast, the gene expression levels of dinoflagellate during coral endosymbiosis have not been investigated due to a lack of transcriptome data 
for the non-symbiotic state. Since the coral-alga model system facilitates the investigation of dinoflagellate gene expression and proliferation processes over time, it is well suited for examining changes in dinoflagellate during endosymbiosis.

In this study, we obtained transcriptome data for cultured Durusdinium that were previously used in an infection experiment with juvenile corals [14]. Therefore, in order to comprehensively investigate the changes in Symbiodiniaceae associated with the transition to the symbiotic state, we attempted to detect differentially expressed genes between the free-living and endosymbiotic states. Following the inoculation of juvenile corals with Durusdinium, its rate of increase was greater than that of Cladocopium, with about 300 Durusdinium cells per polyp detected by the 10th day of endosymbiosis, and about 600 cells per polyp detected by the 20th [14]. Here, we identified and functionally annotated differentially expressed genes (DEGs) between the non-symbiotic and symbiotic states, and performed phylogenetic analyses for a part of the DEGs to confirm that the DEGs were derived from Durusdinium.

\section{Materials and Methods}

Symbiodiniaceae strains CCMP 2556 (genus Durusdinium) were purchased from the Bigelow Laboratory for Ocean Sciences (West Boothbay Harbor, ME, USA; https: / ccmp. bigelow.org/ (accessed on 20 Octorber 2011)). Cultures were grown at $24{ }^{\circ} \mathrm{C}$ under a $12 \mathrm{~h} / 12 \mathrm{~h}$ light/dark cycle at $80 \mu \mathrm{mol} \mathrm{m}^{-2} \mathrm{~s}^{-1}$, in $100 \mathrm{~mL}$ of filtered seawater. Further details on RNA isolation and sequencing are provided in supplementary data.

Illumina HiSeq2000 transcriptome data for the symbiotic state of Durusdinium trenchii were obtained from the DDBJ Sequence Read Archive (accession nos. DRR119964-119967). Data obtained 10 and 20 days after coral incubation with $D$. trenchii [14], and those obtained in the present study, were used for DEG analysis. The quality checking of filtered reads, mapping, and the detection of DEGs between the non-symbiotic and symbiotic states were performed as described by Yuyama et al., 2018, and the supplementary materials. To confirm the calculated RNA-seq results, we performed bootstrap resampling of the raw read data, with 100 replicates per sample using the isoDE2 package [17], and with 100 replicates $(n=10)$ for each free-living or symbiotic sample (duplicate) in order to examine the expression changes between these states. Since the ANOVA using 100 bootstrap resampling results showed considerable variation among replicates for the data collected at day 10 of endosymbiosis, we eliminated these data from our analysis in order to detect changes related to endosymbiosis. Student's $t$-test using 100 resampling results generated undetectably low $p$-values for most genes, so 10 items of data were randomly selected from the 100 replicates (100 bootstrap resampling replicates) and a $t$-test was performed. Differences in the mean (among the 100 replicates) expression of each gene between the two states were detected using the $t$-test $(p<0.025)$. The $p$-value was set to correspond to the number of DEGs detected in edgeR analysis $(q=0.01)$. These obtained DEGs were compared with those detected via the TCC analysis, and those in common were selected. We also selected genes with an expression change of $\log _{2}$ (fold change) $>1$ between states. The functional annotations of the DEGs are described in the supplementary materials.

Among the DEGs, we focused on dapdiamide A synthetase genes characteristic of Dursdinium. A phylogenetic analysis of dapdiamide A synthase was performed using the homologous gene sequences derived from diverse organisms in order to confirm that the genes were derived from Durusdinium rather than from bacteria. Further details on the phylogenetic analysis are given in the supplementary materials.

\section{Results and Discussion}

In this study, we attempted to clarify the gene expression changes taking place in dinoflagellate during coral-alga endosymbiosis establishment. We prepared and sequenced a cDNA library derived from the symbiont culture, which isolated 25,068 contigs containing ORFs. Low reads derived from algae engaged in endosymbiosis with corals and free-living cultured algae were mapped against these contigs, and DEGs between these states were 
identified. The edgeR analysis identified 8543 DEGs, representing $34 \%$ of the candidate alga-derived transcripts. To validate these results, we used bootstrap replicates of RNAseq data to detect DEGs between the non-symbiotic $(n=2)$ and symbiotic states $(n=2)$. Differences in the mean (among $100 \times 2$ replicates) expression of each gene between the two states were detected using the Student's $t$-test $(p<0.025)$. The $p$ value was set to correspond to the number (8642) of DEGs detected in the edgeR analysis $(q=0.01)$. A total of 4587 DEGs were common between both groups. Finally, we selected 1214 genes with $\log _{2}$ (fold change) $>1$ between the free-living and symbiotic states in the edgeR analysis (Figure S1). The top 50 genes showing expression changes due to endosymbiosis with the lowest FDR included ribosomal proteins, heat-shock proteins, and chlorophyll-binding proteins (Figure S1). We also searched for GO molecular function terms that were enriched in these 1214 genes (Figure S2). The most enriched GO terms for these upregulated genes included protein-chromophore linkage and photosynthesis. The upregulated DEGs indicated that algae have enhanced photosynthetic activity during endosymbiosis with corals, which is consistent with previous reports of endosymbiosis in Symbiodiniaceae [7]. Seven processes were related to downregulated DEGs, including microtubule-based processes, mRNA splicing via spliceosome, and protein folding. Genes with decreased expression in microtubule-based processes include genes encoding tubulin, which is a component of flagella. This result may reflect the fact that algae lose their flagella inside corals [18]. Furthermore, a large number of ribosomal and chaperone proteins were detected among the downregulated DEGs, suggesting that some translational and protein-folding functions were inactivated following endosymbiosis establishment. Decreased expression of the chaperone gene has also been reported in the genera Symbiodinium and Cladocopium [11], and may represent a typical response to coral endosymbiosis establishment. It should be noted, however, that some of the DEGs detected include genes that were altered due to environmental differences between the two states. The time of year when the symbiotic and non-symbiotic states were cultured, as well as changes in the light environment, salinity, and $\mathrm{CO}_{2}$ in the coral cells, may have affected the genes whose expressions were altered. In order to investigate more specific changes in a symbiotic organism, we must more closely replicate the exact conditions of the culture strain and the symbiotic state.

Among the top 100 DEGs, two genes encoding dapdiamide A synthase (TRINITY_DN38519_c0_g1_i5.p1 (Figure 1) and TRINITY_DN38519_c0_g1_i1.p1) were found to be upregulated. Dapdiamide A synthase adds valine to the carboxylate of fumaramoylDAP to form dapdiamide A, an antibiotic, in Pantoea agglomerans [19]. Few studies have reported on the antibiotic synthase in Symbiodiniaceae; however, a recent large-scale transcriptome analysis identified dapdiamide A synthase in Symbiodinium [20]. Therefore, we performed a phylogenetic analysis to investigate whether the gene encoding dapdiamide A synthase is derived from Symbiodiniacea or from bacteria (Figure 2). One of the genes (TRINITY_DN38519_c0_g1_i5.p1) encoding dapdiamide A synthase was used for a BLASTp query against the NCBI database, and 117 sequences were selected for phylogenetic inference. The distribution of eukaryotic dapdiamide A synthase was restricted to large phylogenetic groups including stramenopiles, haptophytes, and alveolates (Symbiodiniaceae). In the ML tree (Figure 2), most of the eukaryotic sequences formed two separate clades, A and B. Clade A comprises sequences derived from bacillariophytes (stramenopiles), haptophytes, and Symbiodiniaceae. Nine Symbiodiniaceae sequences were monophyletic, with $86 \%$ support, and its sister group was shared by Emiliania huxleyi (haptophyte) sequences. These branching patterns suggest that the eukaryote-eukaryote lateral gene transfer of dapdiamide A synthase occurred between Emiliania and Symbiodiniaceae. In addition, close relationships between two Symbiodinium sequences as well as archaean (100\%) and Aureococcus (pelagophyte) (no support) sequences on the lower part of the tree suggest other types of lateral gene transfer involving Symbiodiniaceae. However, these genes were detected only in the genera Symbiodinium and Durusdinium of Symbiodiniaceae in this study. Durusdinium species exhibit high stress resistance, and have been reported to confer this property to their host corals [21]. Our results show that both 
dapdiamide A synthase genes from Durusdinium were upregulated during endosymbiosis establishment, which may enhance antibacterial action and confer stress tolerance to the host coral.

replicate 1 replicate 2

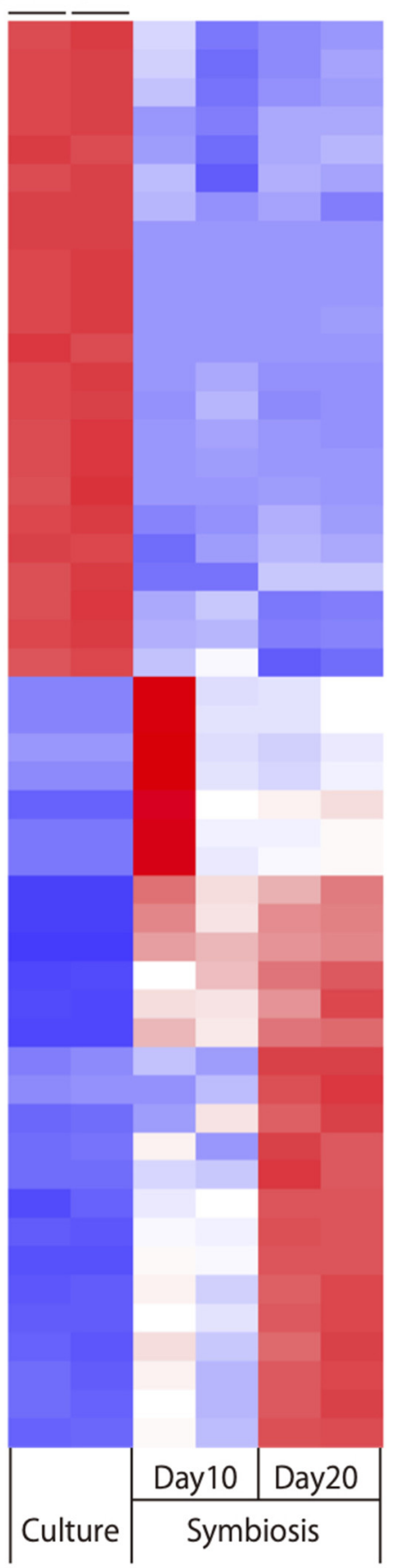

Color Key (Z-score)

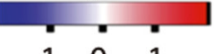

$\begin{array}{lll}-1 & 0 & 1\end{array}$

CAD protein TRINITY_DN37869_c2_99_11.p1

Elongation factor 2 TRINITY DN38361 c1 g1 i1.p1 Elongation factor 2 TRINITY_DN38361_c1_g1_i2.p1 Exoglucanase 1 TRINITY_DN37669_c0_g3_i1.p1

Caroteno-chlorophyll a-c-binding protein (Fragment) TRINITY_DN38778_c2_a1_i12.p1

Heat shock protein 90 TRINITY_DN36968_c1_92_11.p2

Acetyl-CoA carboxylase TRINITY_DN38475_c0 $\mathrm{g} 1$ _ i3.p1 Ribosomal protein rpl-36.A TRINITY_DN77422_C0_91_i1.p2 60S ribosomal protein L27a TRINITY_DN74595_C0_g1_i1.p2 40 S ribosomal protein S5 TRINITY_DN40908_c0_91_i1.p1 60 S ribosomal protein L26-2 TRINITY_DN35825_C0_g2_i1.p1 40S ribosomal protein S13 TRINITY_DN32486_c1_g1_i2.p2 Elongation factor 1-alpha TRINITY_DN37768_c0_-99_11.p2 Elongation factor 1-alpha TRINITY_DN38025_c0_g2_i1.p2 Adenine/guanine permease AZG1 TRINITY_DN35277_c0 g 4 _ i1.p1 Actin, cytoskeletal TRINITY_DN38149_c0_g1_i3.p2 Ubiquitin-40S ribosomal protein S27a TRINITY_DN38015_c0 g10_i2.p2 Nucleolin 1 TRINITY_DN37185_c2_-91_i2.p1 Cytochrome b TRINITY_DN38493_C3_g1_i13.p1 Heat shock $70 \mathrm{KDa}$ protein TRINITY_DN38656_c1_a5 i3.p1 Actin, plasmodial isoform TRINITY_DN38610_c0_g1_i9.p1 Actin TRINITY_DN38149_c0_g2_i1.p1 Actin-3 TRINITY_DN38149_c0_91_i2.p2 Photosystem I P700 chlorophyll a apoprotein A1 TRINITY_DN36521_c0_91_i1.p1 ATP synthase subunit beta, chloroplastic TRINITY_DN24786_c0_91_i1.p1 ATP synthase subunit alpha, chloroplastic TRINITY_DN37518_c1_93_11.p1 Photosystem II CP47 reaction center protein TRINITY_DN37518_c1_ 95 _1 1.p1 Photosystem II D2 protein TRINITY_DN4483_c0_91_i1.p1

Photosystem II CP43 reaction center protein TRINITY_DN37518_c1_97_11.p1 Photosystem I P700 chlorophyll a apoprotein A2 TRINITY_DN37350_c1_91_i1.p1 Photosystem II protein D1 TRINITY_DN90189_C0_91_11.p1 Tartrate-resistant acid phosphatase type 5 TRINITY_DN37137_c2_95_i1.p1 Radial spoke head protein 6 homolog A TRINITY_DN23280_c0_91_i1.p1 DEAD-box ATP-dependent RNA helicase 28 TRINITY_DN36912_C0_g2_14.p1 GPI mannosyitransferase 3 TRINITY_DN21168_c0_91_i1.p1 Gamma-aminobutyric acid type B receptor subunit 1 TRINITY_DN32507_c0_91_i1.p1 Sugar phosphate exchanger 3 TRINITY_DN15572_c0_g1_i1.p1 YEATS domain-containing protein 2 TRINITY_DN38009_c0_92_i1.p1 Cytochrome c6 TRINITY_DN38547_c1_92_i1.p2 Dapdiamide A synthase TRINITY_DN38519_C0_91_i1.p1 Fibrocystin-L TRINITY_DN37062_c0_g2_i1.p1 Cytochrome b6-f complex subunit 5 TRINITY_DN27935_c0_91_i3.p1

Fucoxanthin-chlorophyll a-c binding protein F, chloroplastic (Fragment) TRINITY_DN38274_c0_91_i3.p1 Fucoxanthin-chlorophyll a-c binding protein F, chloroplastic (Fragment) TRINITY_DN38274_c0_-91_11.p1

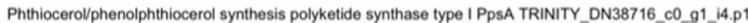
Non-reducing polyketide synthase mapC TRINITY_DN38716_c0_g1_i5.p1 Acetylcholine receptor subunit epsilon TRINITY_DN36714_c0_91_i3.p1

Protein disulfide-isomerase TRINITY_DN38201_c1_g3_i1.p1 PhthioceroUphenolphthiocerol synthesis polyketide synthase type I PPSA TRINITY_DN38716_c0_91_i1.p1 Dapdiamide A synthase TRINITY_DN38519_c0_91_i5.p1

Figure 1. Heat map of RNA-seq analysis for 50 selected genes showing different gene expression pattern in free-living and symbiotic Durusdinium at 10 and 20 days post-inoculation $(n=2)$. Among the 1214 DEGs shown in Figure S1, the 50 genes with the lowest false discovery rate are summarized in the heatmap. 


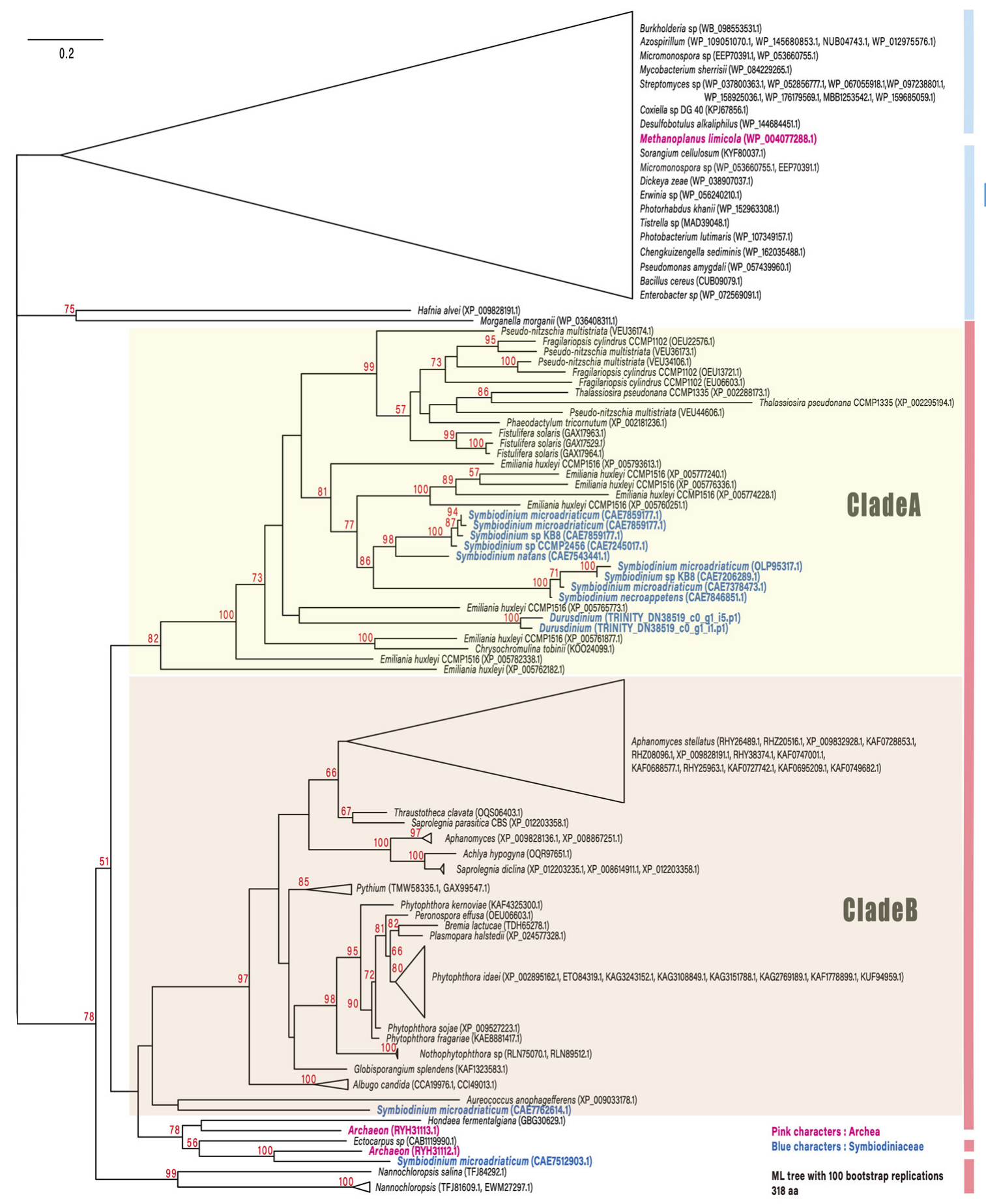

Figure 2. Maximum likelihood phylogenetic tree describing the relationships among dapdiamide A synthase proteins from representative eukaryotes and prokaryotes. All bacterial and one archaean sequence were separated from the remaining eukaryotic/archaeal sequences (78\%). Most of the eukaryotic sequences formed two separate clades, A and B. In clade A, bacillariophyte sequences were monophyletic (99\%), excluding haptophyte and Symbiodiniaceae sequences. Nine Symbiodinium sequences were monophyletic, with $86 \%$ support, and its sister group was shared by Emiliania huxleyi (haptophyte) sequences. Numbers in red near the nodes are ultrafast bootstrap support values; values $<50 \%$ are not included. Blue indicates genes derived from Symbiodiniaceae.

In this study, Durusdinium genes that exhibited expression changes during coral endosymbiosis establishment were selected using two analysis methods for functional analysis. The weakness of this study is the small number of replicas and the different timings of the fixation of symbiotic and non-symbiotic dinoflagellate. In future gene 
expression research, it will be necessary to improve these areas. In addition, transcriptome data do not necessarily correlate with protein expression data, thus requiring proteome analysis to elucidate the entire internal symbiotic process. The roles of these genes in dinoflagellate adaptation to the host coral environment need to be further investigated; such data could be useful in clarifying the evolutionary process of symbiont trait acquisition.

Supplementary Materials: The following are available online at https:/ / www.mdpi.com/article/ 10.3390/microorganisms9081560/s1, Additional information on methods, Figure S1: Number of differentially expressed genes (DEGs) identified by comparing non-symbiotic and symbiotic Durusdinium using edgeR method and Student's $t$-test with bootstrap resampling data, Figure S2: The results of GO (gene ontology) analysis using downregulated 1025 DEG and upregulated 189 DEG against all Durusdinium mRNA sequence isolated our studies.

Author Contributions: Conceptualization, I.Y.; methodology, I.Y. and T.H.; validation, N.U., I.Y., and T.H.; formal analysis, N.U.; investigation, N.U. and I.Y.; resources, I.Y.; data curation, N.U. and I.Y.; writing - review and editing, I.Y. and T.H.; visualization, I.Y.; funding acquisition, I.Y. All authors have read and agreed to the published version of the manuscript.

Funding: This work was supported by a research grant from the Japan Society for the Promotion of Science (\#19H03026), and the Environment Research and Technology Development Fund (No. 4-1806) from the Ministry of the Environment in Japan, and the Kurita Water and Environment Foundation (18B083).

Institutional Review Board Statement: Not applicable.

Informed Consent Statement: Not applicable.

Data Availability Statement: The raw fastq files for the RNA-seq libraries were deposited at $D D B J$ Sequence Read Archive (DRA) with the accession number of DRA10343.

Acknowledgments: We would like to thank the members of the Laboratory of Molecular Evolution of Microbes in the University of Tsukuba for valuable discussion. RNA-seq analyses were partially performed on the NIG supercomputer at the ROIS National Institute of Genetics.

Conflicts of Interest: The authors declare no conflict of interest. The funders had no role in the design of the study; in the collection, analyses, or interpretation of data; in the writing of the manuscript; or in the decision to publish the results.

\section{References}

1. LaJeunesse, T.C.; Parkinson, J.E.; Gabrielson, P.W.; Jeong, H.J.; Reimer, J.D.; Voolstra, C.R.; Santos, S.R. Systematic revision of Symbiodiniaceae highlights the antiquity and diversity of coral endosymbionts. Curr. Biol. 2018, 28, 2570-2580.e6. [CrossRef] [PubMed]

2. Pochon, X.; Gates, R.D. A New Symbiodinium clade (Dinophyceae) from soritid foraminifera in Hawai'i. Mol. Phylogenet. Evol. 2010, 56, 492-497. [CrossRef]

3. Pochon, X.; Montoya-Burgos, J.I.; Stadelmann, B.; Pawlowski, J. Molecular phylogeny, evolutionary rates, and divergence timing of the symbiotic dinoflagellate genus Symbiodinium. Mol. Phylogenet. Evol. 2006, 38, 20-30. [CrossRef]

4. Tanaka, Y.; Miyajima, T.; Koike, I.; Hayashibara, T.; Ogawa, H. Translocation and conservation of organic nitrogen within the coral-zooxanthella symbiotic system of Acropora pulchra, as demonstrated by dual isotope-labeling techniques. J. Exp. Mar. Biol. Ecol. 2006, 336, 110-119. [CrossRef]

5. Whitehead, L.F.; Douglas, A.E. Metabolite comparisons and the identity of nutrients translocated from symbiotic algae to an animal host. J. Exp. Biol. 2003, 206, 3149-3157. [CrossRef]

6. Wang, J.T.; Douglas, A.E. Essential amino acid synthesis and nitrogen recycling in an alga-invertebrate symbiosis. Mar. Biol. 1999, 135, 219-222. [CrossRef]

7. Barott, K.L.; Venn, A.A.; Perez, S.O.; Tambutté, S.; Tresguerres, M. Coral host cells acidify symbiotic algal microenvironment to promote photosynthesis. Proc. Natl. Acad. Sci. USA 2015, 112, 607-612. [CrossRef]

8. Takeuchi, R.; Jimbo, M.; Tanimoto, F.; Iijima, M.; Yamashita, H.; Suzuki, G.; Harii, S.; Nakano, Y.; Yasumoto, K.; Watabe, S. N-Acetyl-D-Glucosamine-binding lectin in Acropora tenuis attracts specific Symbiodiniaceae cell culture strains. Mar. Drugs 2021, 19, 146. [CrossRef]

9. Kuniya, N.; Jimbo, M.; Tanimoto, F.; Yamashita, H.; Koike, K.; Harii, S.; Nakano, Y.; Iwao, K.; Yasumoto, K.; Watabe, S. Possible involvement of Tachylectin-2-like lectin from Acropora tenuis in the process of Symbiodinium Acquisition. Fish. Sci. 2015, 81, 473-483. [CrossRef] 
10. Maor-Landaw, K.; van Oppen, M.J.H.; McFadden, G.I. Symbiotic lifestyle triggers drastic changes in the gene expression of the algal endosymbiont Breviolum minutum (Symbiodiniaceae). Ecol. Evol. 2020, 10, 451-466. [CrossRef] [PubMed]

11. Mohamed, A.R.; Andrade, N.; Moya, A.; Chan, C.X.; Negri, A.P.; Bourne, D.G.; Ying, H.; Ball, E.E.; Miller, D.J. Dual RNAsequencing analyses of a coral and its native symbiont during the establishment of symbiosis. Mol. Ecol. 2020, 29, $3921-3937$. [CrossRef]

12. Bertucci, A.; Tambutté, É.; Tambutté, S.; Allemand, D.; Zoccola, D. Symbiosis-dependent gene expression in coral-dinoflagellate association: Cloning and characterization of a P-Type H+-ATPase gene. Proc. R. Soc. B Biol. Sci. 2010, 277, 87-95. [CrossRef] [PubMed]

13. Watanabe, T.; Kii, S.; Tanaka, J.; Takishita, K.; Maruyama, T. cDNA cloning and phylogenetic and expression analyses of actin in symbiotic dinoflagellates (Symbiodinium spp.). J. Appl. Phycol. 2006, 18, 219-225. [CrossRef]

14. Yuyama, I.; Ishikawa, M.; Nozawa, M.; Yoshida, M.; Ikeo, K. Transcriptomic changes with increasing algal symbiont reveal the detailed process underlying establishment of coral-algal symbiosis. Sci. Rep. 2018, 8, 16802. [CrossRef]

15. Yuyama, I.; Higuchi, T. Comparing the effects of symbiotic algae (Symbiodinium) clades C1 and D on Early growth stages of Acropora tenuis. PLoS ONE 2014, 9, e98999. [CrossRef] [PubMed]

16. Yoshioka, Y.; Yamashita, H.; Suzuki, G.; Zayasu, Y.; Tada, I.; Kanda, M.; Satoh, N.; Shoguchi, E.; Shinzato, C. Whole-genome transcriptome analyses of native symbionts reveal host coral genomic novelties for establishing coral-algae symbioses. Genome Biol. Evol. 2021, 13, evaa240. [CrossRef]

17. Al Seesi, S.; Tiagueu, Y.T.; Zelikovsky, A.; Măndoiu, I.I. Bootstrap-based differential gene expression analysis for RNA-Seq data with and without replicates. BMC Genom. 2014, 15, S2. [CrossRef] [PubMed]

18. Camaya, A.P. Stages of the symbiotic zooxanthellae-host cell division and the dynamic role of coral nucleus in the partitioning process: A novel observation elucidated by electron microscopy. Coral Reefs 2020, 39, 929-938. [CrossRef]

19. Hollenhorst, M.A.; Clardy, J.; Walsh, C.T. The ATP-dependent amide aigases DdaG and DdaF assemble the fumaramoyl-dipeptide scaffold of the Dapdiamide antibiotics. Biochemistry 2009, 48, 10467-10472. [CrossRef]

20. Silva Lima, A.W.; Leomil, L.; Oliveira, L.; Varasteh, T.; Thompson, J.R.; Medina, M.; Thompson, C.C.; Thompson, F.L. Insights on the genetic repertoire of the coral Mussismilia braziliensis endosymbiont Symbiodinium. Symbiosis 2020, 80, 183-193. [CrossRef]

21. Stat, M.; Gates, R.D. Clade D Symbiodinium in Scleractinian Corals: A “Nugget" of Hope, a Selfish Opportunist, an Ominous Sign, or All of the Above? J. Mar. Biol. 2010, 2011, e730715. [CrossRef] 